\title{
Novel Single Gold Nanowire-based Electrochemical Immunosensor for Rapid Detection of Bovine Viral Diarrhoea Antibodies in Serum
}

\author{
Armelle Montrose ${ }^{1}$, Niamh Creedon', Ryona Sayers ${ }^{2}$, Sean Barry ${ }^{1}$ and Alan O'riordan ${ }^{1 *}$ \\ ${ }^{1}$ Tyndall National Institute, University College Cork, Dyke Parade, Cork, Ireland \\ ${ }^{2}$ Teagasc, Moorepark, Fermoy, Cork Ireland
}

\begin{abstract}
Bovine Viral Diarrheoa (BVD) is a worldwide disease with severe financial implications for the Bovine beef and dairy industries. A key challenge to BVD eradication is that the requirement to send samples to, and receive results from, specialized laboratories slows down the diagnostic process and leads to uncontrolled spread of the virus within a herd until diagnostic confirmation is received. Consequently, rapid identification of BVD is now critical for herd protection and prevention of costly herd outbreaks and new diagnostic devices, suitable for on-farm analysis, that deliver rapid and early identification of animal disease states, are required. We report, here, an electrochemical onchip fully integrated nanowire based immunosensor device for detection of BVD in serum. The capture biomolecule, BVD virus, is covalently immobilized via a carboxylic terminated polymer firstly electrodeposited onto a single nanowire. Electrochemical characterization including faradaic electrochemical impedance spectroscopy and cyclic voltammetry is performed. Label free immunologic detection of antibodies $(10 \mu \mathrm{g} / \mathrm{mL}, 20 \mathrm{~min})$ is first demonstrated using a bovine serum albumin as a model antigen-antibody system. Then, the immunosensor is applied to detection of bovine viral diarrhoea antibodies $(10 \mu \mathrm{g} / \mathrm{mL}, 20 \mathrm{~min})$ in both buffer and serum. The sensor clearly discriminates between positive and negative infected bovine sera. This study clearly shows the potential of this chip nanowirebased electrochemical sensor for immunoassays application in serum with a view to developing portable devices for on-farm diagnosis or therapeutic monitoring in animal health applications.
\end{abstract}

Keywords: Immunosensor; Nanowire; Electrochemical impedance spectroscopy; Electrodeposited polymer; Bovine viral diarrhoea

\section{Introduction}

Bovine Viral Diarrhoea (BVD), is a major contributor to bovine respiratory disease and one of the most common diseases in cattle $[1,2]$. BVD virus is a pestivirus in the Flaviviridae family and has a worldwide distribution. Once BVD virus enters a herd, viral spread is rapid and full herd-infection can result within weeks. Economic implications of an outbreak are severe as diseased animals perform sub-optimally and decrease on-farm efficiency and profitability through waste feed, labor and increased veterinary costs. Delayed diagnosis of viral infections in animals can also lead to animal death.

European farmers $€ 300$ per cow in treatment/isolation costs [3]. Likewise, in the US this disease has a total economic cost to the cattle industry of over US $\$ 2$ billion annually. Early identification of respiratory diseases are essential to achieve effective control, in that, appropriate intervention with removal from the herd or vaccines treatments can reduce clinical signs and reduce the number of carrier animals present within a herd [3]. To date, only a few European countries have achieved total eradication and in many countries national schemes have not been initiated as they are deemed too costprohibitive and time-consuming.

Detection of BVD is currently performed using high-end analytical instrumentation including: Reverse Transcription Polymerase Chain Reaction (RT-PCR), immunohistochemistry or Enzyme Linked Immunosorbant Assay (ELISA) by targeting whole BVD virus, specific antigen epitopes or specific antibodies [4-6]. Globally, these tests have sufficient sensitivity but can be time-consuming (a few hours). They also require dedicated laboratories, skilled personnel and have high instrument ownership and maintenance costs. Rapid detection and identification of BVD virus is critical for herd protection in order to prevent costly herd outbreaks. However current diagnostic approaches (based on laboratory-based methods) can slow down this diagnostic process as samples must be first sent to the designated laboratory; once received and analyzed results must then be communicated back to the key stakeholders (farmers and veterinarians), a process that can take days to weeks. These delays can lead to uncontrolled spread of the virus within a herd. Consequently there is now a growing need to develop new rapid diagnostic tools that will deliver rapid and early identification ( $<30$ minutes) of animal disease state on-farm.

Electrochemical biosensors, though label-free detection methods, constitute a promising group of sensing devices that allow high sensitivity, short analysis times, affordability, miniaturized platforms, with low sample consumption and the possibility for measurements in complex samples [7]. Amperometry, potentiometry and electrochemical impedance spectroscopy (EIS) are examples of electrochemical techniques that have been used in immunosensors [8]. Of these, EIS represents a powerful method for the detection of target analyte at functionalized sensor surface via an immunochemical interaction in a label free manner, i.e., without the need for further costly or complex labeling techniques [9-11]. EIS uses periodic small AC voltage amplitudes that are applied to an electrode and a change of the electrode impedance is measured and can be correlated to the amount of analyte binding $[12,13]$.

*Corresponding author: Alan O'riordan, Tyndall National Institute, University College Cork, Dyke Parade, Cork, Ireland, Tel: 35321490 30; E-mail: alan.oriordan@tyndall.ie

Received March 30, 2015; Accepted July 29, 2015; Published September 01, 2015

Citation: Montrose A, Creedon N, Sayers R, Barry S, O'riordan A (2015) Nove Single Gold Nanowire-based Electrochemical Immunosensor for Rapid Detection of Bovine Viral Diarrhoea Antibodies in Serum. J Biosens Bioelectron 6: 174 doi:10.4172/2155-6210.1000174

Copyright: @ 2015 Montrose A, et al. This is an open-access article distributed under the terms of the Creative Commons Attribution License, which permits unrestricted use, distribution, and reproduction in any medium, provided the original author and source are credited. 
Recent developments in fabrication of robust and reproducible nanoelectrodes have opened the door to a new and exciting area of electrochemistry. Compared to traditional macroelectrodes, nanoscale electrodes, such as those based on single nanowires, have tremendous potential when employed in electrochemical analysis due to enhanced sensitivity. This enhancement arises from increased mass transport to the electrode (via radial diffusion) and low background charging currents (due to reduced surface area) relative to macroscale electrodes [10,14]. Reproducible wafer-scale fabrication (using nanoelectronic fabrication techniques) of fully integrated nanowire-based electrochemical devices have been previously developed $[15,16]$ and the applicability of these devices for highly sensitive detection of (bio) molecules including: glucose and hydrogen peroxide [17], heavy metals [18] and explosives [19] have been demonstrated. These discrete nanowire sensor devices exhibit a 100-10,000 fold increase (analyte dependent) in sensitivity and can undertake analysis in milliseconds compared to minutes for commercial state-of-the-art electrodes [20,21].

To date, field effect transistor based semiconductor nanowire sensors have been successfully employed for detection of key biomolecules including DNA, viruses and disease biomarkers [22-24]. Although elegant, these approaches are limited in that they require application of a high back gate voltage (up to $40 \mathrm{~V}$ DC), and many of their attributes such as reproducibility, product uniformity, potential for scaling up and cost effectiveness are not very well known [25]. Combining electrochemical impedance techniques with highly reproducible gold nanoelectrodes could, therefore, enable the development of new upstream diagnostic devices. However, a critical challenge to developing such devices is that label-free electrochemical based detection of biomolecule binding events at the discrete nanostructures in buffer or bio-media has yet to be demonstrated.

In this paper, we address this challenge and demonstrate the first on-chip nanowire immunosensor device for the electrochemicalbased detection of BVD antibodies in serum. Nanowires are fabricated using reproducible top-down nanofabrication approaches used in the nanoelectronics industry. Capture biomolecules are covalently immobilized onto an electrodeposited polymer layer pre-deposited on a nanowire sensor and each step of the modifying process is characterized using cyclic voltammetry and EIS. Immunoassays are developed using a bovine serum albumin antibody/antigen model system. The immunosensor is first applied to the detection of anti-BSA antibodies in buffer and then to BVD antibodies in both buffer and diluted serum. Finally the sensor is challenged to discriminate between disease positive and disease negative serum samples. BVD assay times are typically $\sim 20$ minutes demonstrating the potential of these nanowire electrochemical immunosensors for use in future portable devices required for on-farm diagnostic applications.

\section{Identification of the agent}

BVDV is a pestivirus in the family Flaviviridae and is closely related to classical swine fever and ovine border disease viruses. The two genotypes (types 1 and 2) are classified as separate species in the genus Pestivirus. A third putative genotype, BVDV type 3 , has also recently been proposed. Although both cytopathic and non-cytopathic biotypes of BVDV type 1 and type 2 exist, non-cytopathic strains are usually encountered in field infections and are the main focus of diagnostic virus isolation in cell cultures. PI animals can be readily identified by a variety of methods aimed to detect viral antigens or viral RNA directly in blood and tissues. Virus can also be isolated by inoculation of specimens onto susceptible cell cultures followed by immunelabelling methods to detect the replication of the virus in the cultures.
Persistence of virus infection should be confirmed by resampling after an interval of at least 3 weeks, when virus will again be detected. PI animals are usually seronegative. Viraemia in acute cases is transient and difficult to detect. Virus isolation in semen from bulls that are acutely or persistently infected requires special attention to specimen transport and testing. RNA detection assays are particularly useful because they are rapid, have very high sensitivity and do not depend on the use of cell cultures.

\section{Serological tests}

Acute infection with BVDV is best confirmed by demonstrating seroconversion using sequential paired samples, ideally from several animals in the group. The testing of paired (acute and convalescent samples) should be done a minimum of 21 days apart and samples should be tested concurrently in the same assay. Enzyme-linked immunosorbent assays and the virus neutralisation test are the most widely used.

\section{Requirements for vaccines}

There is no standard vaccine for BVD, but a number of commercial preparations are available. An ideal vaccine should be able to prevent transplacental infection in pregnant cows. Modified live virus vaccine should not be administered to pregnant cattle (or to their sucking calves) due to the risk of transplacental infection. Live vaccines that contain cytopathic strains of BVDV present a risk of inducing mucosal disease in PI animals. Inactivated viral vaccines are safe and can be given to any class of animal but generally require booster vaccinations. BVDV is a particularly important hazard to the manufacture of vaccines and biological products for other diseases due to the high frequency of contamination of batches of fetal calf serum used as a culture medium supplement.

\section{Materials and Methods}

\section{Materials}

O-aminobenzoic acid (o-ABA), N-hydroxysuccinimide (NHS), $\mathrm{N}$-ethyl-N-(dimethylaminopropyl)-carbodiimide (EDC), phosphate buffered saline (PBS) solution, Dubelcco's phosphate buffered saline $10 \times(\mathrm{PBS} 10)$ solution, tween 20 , sodium chloride $(\mathrm{NaCl})$, sodium hydroxide $(\mathrm{NaOH})$, bovine serum albumin (BSA), anti-bovine albumin antibody (BSA $\mathrm{Ab})$, and ferrocene monocarboxylic $(\mathrm{FcCOOH})$ were purchased from Sigma-Aldrich. The acetate buffer (10 mM; pH 4) and ethanolamine- $\mathrm{HCl}(1 \mathrm{mM})$ were obtained from Sierra Sensors $\mathrm{GmbH}$ (Germany). Deionized water $(18.2 \mathrm{M} \Omega \mathrm{cm}$ ) was obtained using an ELGA Pure Lab Ultra system. BVD virus and monoclonal antibodies BVD specific to the envelop glycoprotein (Erns) of the virus were purchased from Animal and Plant Health Agency (APHA Scientific, UK). All reagents were used as received. Positive and negative bovine serum samples were provided by Teagascbiobank (Moorepark, Ireland).

\section{Methods}

Fully integrated nanowire based electrode fabrication: Gold nanowires electrodes, on-chip gold counter and platinum pseudoreference electrodes were fabricated on wafer-scale silicon substrates using hybrid e-beam/optical lithography, metaldeposition and lift-off techniques, as previously described in detail by Wahl et al. [19]. Before use, chips were cleaned using a mixed solvent clean process and dried under a stream of nitrogen.

Electrode modification: A schematic diagram of the immunosensor 
is shown in Figure 1. All the modification steps were performed in a bespoke electrochemical cell. On-chip nanowire electrodes were first modified using electrodeposited o-ABA. The electrodeposition of the polymer (10 mM o-ABA in $10 \mathrm{mM}$ acetate buffer $\mathrm{pH} 4)$ was undertaken by cyclic voltammetry ( 25 cycles) in the applied potential ranging of -0.2 to $0.8 \mathrm{~V}$ (versus on-chip pseudo platinum electrode) at a scan rate of $50 \mathrm{mV} / \mathrm{s}$ followed by careful rinsing of the electrodes with DI water to remove any remaining monomers. The carboxylic terminated polymer was activated using a fresh mixture of EDC/NHS $(10 \mathrm{mM} / 10 \mathrm{mM})$ for $30 \mathrm{~min}$. Then, capture molecules, either BSA $(10 \mathrm{mg} / \mathrm{mL})$ or BVD virus $(100 \mu \mathrm{g} / \mathrm{mL})$, were covalently attached to the surface $\left(3\right.$ hours at $\left.4^{\circ} \mathrm{C}\right)$. Following this immobilization, the electrodes were well rinsed with PBS10 solution containing 0.1\% Tween-20 (PBS10-T), PBS and DI water to remove any unbound proteins and the un-reacted active sites were blocked by immersing in ethanolamine for $30 \mathrm{~min}$.

Finally, the antigen modified electrodes were exposed to known concentrations of antibody solution in PBS or to $1 \%$ diluted serum samples for $20 \mathrm{~min}$ at room temperature. The electrodes were rinsed carefully with PBS10-T, $\mathrm{NaCl}(1 \mathrm{M}), \mathrm{PBS}, \mathrm{NaOH}(10 \mathrm{mM})$ and DI water to remove non-specifically bound target biomolecules prior to measurement.

Electrochemical measurements: Electrochemical experiments were carried out using an Autolab Potentiostat/ Galvanostat PGSTAT128N (Metrohm Ltd, Utrecht, The Netherlands) controlled by the Autolab NOVA software. All experiments employed a standard three-electrode cell configuration using a single gold nanowire as the working electrode, versus the on-chip gold counter electrode and the on-chip platinum pseudo-reference electrode.

Cyclic voltammetry (CV) and faradaic electrochemical impedance spectroscopy (EIS) were performed in a $10 \mathrm{mM}$ PBS solution $(\mathrm{pH}=7.4)$ containing $1 \mathrm{mMFcCOOH}$. For $\mathrm{CV}$, the potential was cycled from -0.2 to $0.6 \mathrm{~V}$ (versus on-chip platinum pseudo reference electrode) at a scan rate of $100 \mathrm{mV} / \mathrm{s}$. The impedance spectra were recorded in a frequency range of $100 \mathrm{mHz}$ to $100 \mathrm{kHz}$ at $150 \mathrm{mV}$, the equilibrium potential of the $\mathrm{FcCOOH}$ redox couple. The amplitude of the alternating voltage

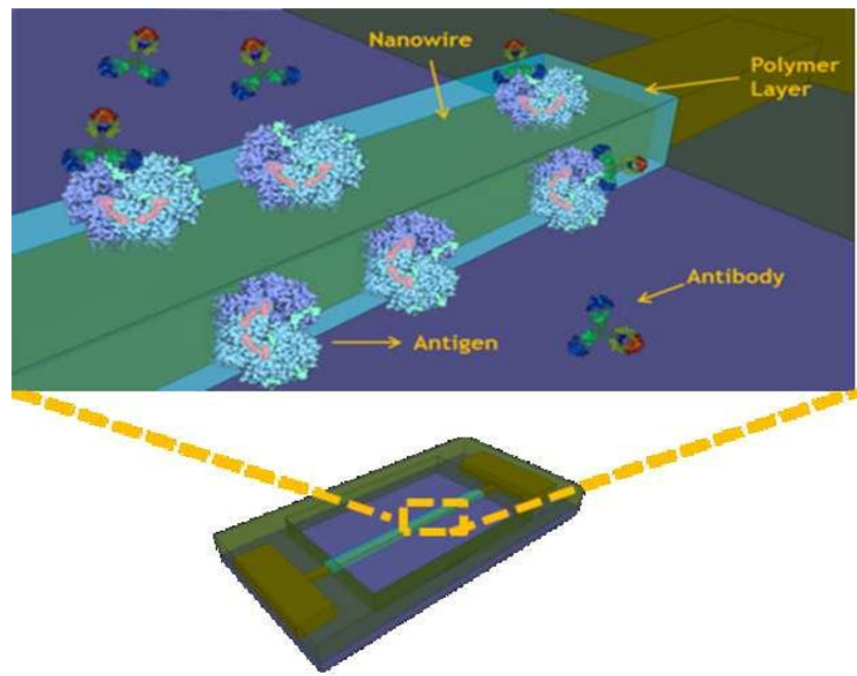

Figure 1: Schematic of an immunoassay at a single gold nanowire. A polyme layer is first electrodeposited at a single nanowire electrode. Capture biomolecule, virus or antigens, are then covalently attached on the electrodeposited polyme layer and used to specifically capture and bind to target antibodies which are subsequently detected electrochemically.



B)

C)

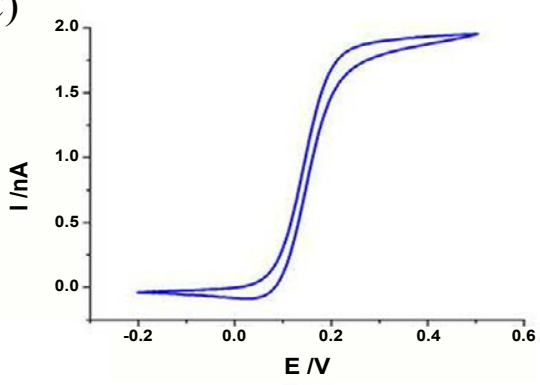

Figure 2: (A) Photograph of the electrochemical cell and the chip containing 12 electrodes and on chip platinum reference and gold counter electrodes; (B) Optical photography of a single gold nanowire of $700 \mathrm{~nm}$ width; (C) Typical Cyclic voltammogram with an applied potential ranging from -0.2 to $0.5 \mathrm{~V}$ obtained for a single nanowire in $1 \mathrm{mMFcCOOH}$ in $10 \mathrm{mM}$ PBS (pH 7.4).

was $5 \mathrm{mV}$. All experiments were performed at room temperature in a Faraday cage.

ELISA measurements: ELISA tests were performed using a commercial BVDV p80 Ab detection kit for the detection of specific antibodies directed to bovine viral diarrhea virus (IDEXX, UK) following the short $(1 \mathrm{~h})$ protocol. Briefly, the p80 modified ELISA plate of the kit was exposed to the serum samples diluted (10\%) in the commercial dilution solution. Following $1 \mathrm{~h}$ incubation at room temperature, plates were rinsed with diluted commercial wash buffer using an ELISA plate washer (DIASource, Belgium). Then the conjugate was diluted (with the provided solution) and incubated in the plate for $30 \mathrm{~min}$ at room temperature. After washing, a chromogenic substrate was added for $20 \mathrm{~min}$ in dark room at room temperature. Finally, the reaction revealing the conjugate was stopped using the commercial stop solution and the absorbance was read at $450 \mathrm{~nm}$ using an ELISA plate reader (DIASource, Belgium). Blank and controls using positive and negative provided control solutions were also performed. All samples were analyzed in triplicate.

\section{Results and Discussion}

\section{Characterization of gold nanowire electrode}

Fabricated devices were mounted in an electrochemical cell setup (Figure 2A) and contain twelve separate electron-beam fields containing nanowire electrodes, an on-chip gold counter electrode, an on-chip platinum pseudo-reference electrode and peripheral contact pads to facilitate direct electrical and electrochemical probing. Nanowire electrodes were $700 \mathrm{~nm}$ width and $45 \mu \mathrm{m}$ long (Figure 2B). Electrochemical functionality of the nanowire electrode was characterized using cyclic voltammetry in presence of a redox probe. Figure $2 \mathrm{C}$ shows a typical voltammogram obtained in $1 \mathrm{mMFcCOOH}$ with an intensity current of $2 \mathrm{nA}$, at a single gold nanowire electrode. As expected, a quasi-steady-state response was observed, typical of single electron oxidation occurring at a discrete nanowire [26]. The magnitude of the current confirms that electrochemistry only occurs at 
the nanowire electrodes and the passivation layer successfully prevents unwanted electrochemistry occurring at on-chip metallisation. Nanowire devices that exhibited lower or no electrochemical current were discarded and not used for further experiments as they revealed a defect in the nanowire or an incomplete removal of the passivation layer.

\section{Application of the biofunctionnalization process to a model BSA antigen-antibody}

Electrodeposition of o-ABA: The modification process is based on the electrodeposition of an o-ABA polymer layer which is then used to immobilize the antigen onto a single gold nanowire electrode. Poly o-aminobenzoic acid is selected since it is a carboxyl functionalized aniline polymer capable of self-doping. This bio-compatible polymer is emerging as a promising polyaniline derivative for biosensor applications. After polymerization, the free carboxylic acid functional groups can be used as a matrix material for subsequent immobilization though covalent binding with biomolecules such as proteins and antibodies [27-31].

A typical CV obtained for the electrodeposition of o-ABA on the single nanowire. The first cycle of the electrodeposition displays an oxidative peak around $0.5 \mathrm{~V}$ corresponding to the formation of the polymer on the gold electrode. This peak disappears with subsequent scans and a small reversible peak around $0 \mathrm{~V}$, characteristic of the doping-dedoping of the polymer, and a second dedoping peak at 0.3 $\mathrm{V}$ in the cathodic scan appear with increasing number of cycles. The emergence of these peaks confirms the electrodeposition of o-ABA on the gold surface [31].

Detection of BSA antibody in buffer: To confirm suitability of on-chip nanowires for immunoassay applications, the immobilization process and the immunologic detection of BSA antibody onto the o-ABA modified nanowire was first demonstrated. Figure 3A shows a schematic of the layer-by-layer build up on a nanowire surface. Each step of the assays was characterized using both cyclic voltammetry and impedance spectroscopy. The CV and the Nyquist spectra for the detection of $10 \mu \mathrm{g} / \mathrm{mL} \mathrm{BSA} \mathrm{Ab}$ are presented in Figures 3B and 3C, respectively.

The CV of the cleaned gold nanowire electrode shows a typical quasi-steady-state response characteristic of the redox couple (Figure $3 \mathrm{~B}$, curve a) and the associated nyquist spectrum (Figure 3C, curve a) displays a semi-circle with an estimated charge transfer resistance around $58 \pm 16 \mathrm{M} \Omega$. After the electrodeposition of o-ABA layer, the current intensity in the $\mathrm{CV}$ decreases (Figure $3 \mathrm{~B}$, curve $\mathrm{b}$ ) while nyquist semi-circle increases (Figure 3C, curve b). This indicates an increase in charge transfer resistance and in the global capacitance at the nanowire. These changes may arise from the electrodeposited polymer partially blocking the electrode and therefore limiting the electron transfer from FcCOOH to the electrode. The negative charges of the carboxylate terminated polymer can also induce an electrostatic repulsion of bulk $\mathrm{FcCOOH}$ ions. Covalent immobilization of the BSA is undertaken though the amide bond formed by the EDC-NHS coupling. No major change is visible on the voltammogram (Figure 3B, curve c) but the nyquist spectrum (Figure 3C, curve c) reveals a decrease in the semicircle. This change could be attributed to the covalent immobilization of BSA at the carboxylate activated sites of the polymer rather than the simple adsorption on the electrode as the accessible surface for the oxidation and reduction of the $\mathrm{FcCOOH}$ remains constant. This decrease in measured impedance could be attributed to a decrease in charge density following binding (arising from displacement of solvent counter ions) and an increase in the thickness of the modifying layer leading to a decrease in the global capacitance at the nanowire. After ethanolamine blocking step, no significant difference (from the BSA curve) is visible in the $\mathrm{CV}$ (Figure $3 \mathrm{~B}$, curve $\mathrm{d}$ ) or the nyquist spectrum (Figure 3C, curve d) suggesting that BSA is bound to all accessible active sites.

For the immunologic detection, anti-BSA antibodies (BSA Ab; $10 \mu \mathrm{g} / \mathrm{mL}$ ) in buffer solution (PBS; $\mathrm{pH} 7.4$ ) are deposited on the fully blocked BSA-modified nanowire and allowed to incubate for $20 \mathrm{~min}$. The binding of BSA Ab leads to a strong decrease in current intensity (Figure 3B, curve e), and an increase in the impedance (Figure $3 \mathrm{C}$,
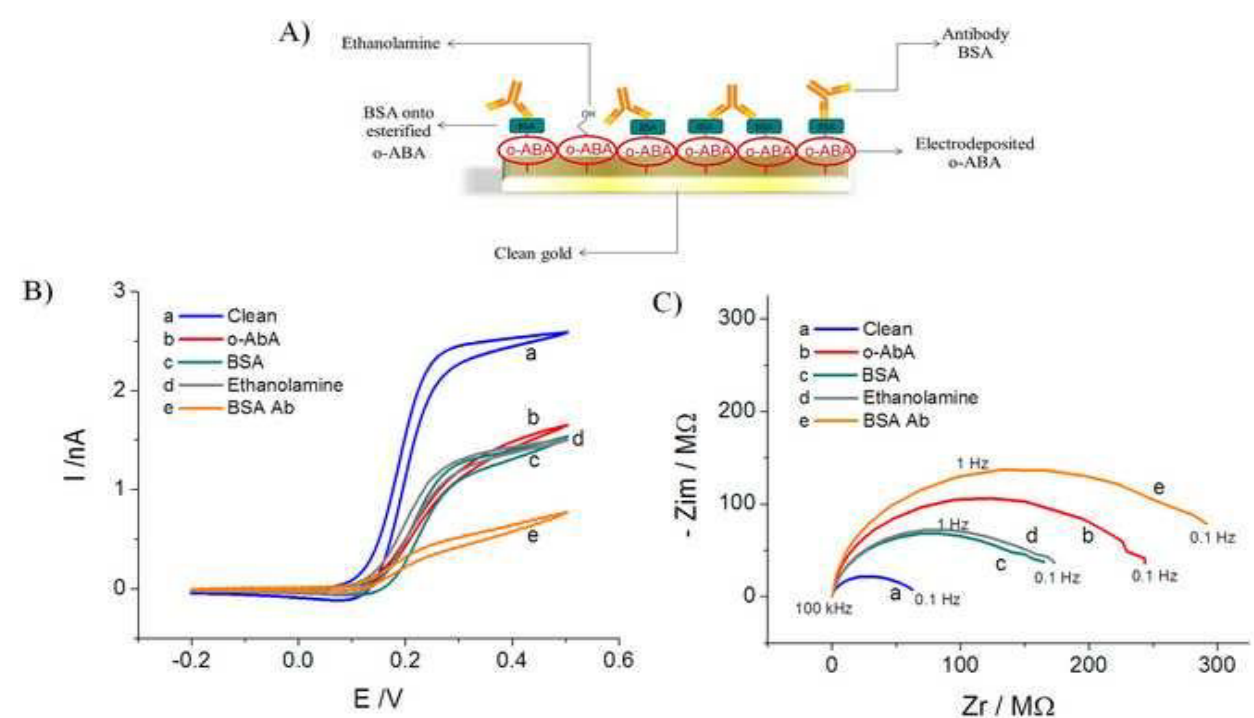

Figure 3: (A) Schematic of the layer-by-layer build up on a nanowire sensor for the BSA antigen/antibody model system; (B) Cyclic Voltammograms and $(C)$ Nyquist plots of (a) pristine gold nanowire electrode;(b) o-ABA modified electrode; (c) BSA (10 mg/mL) modified electrode; (d) ethanolamine blocked electrode; (e) Antibody BSA binding (10 $\mu \mathrm{g} / \mathrm{mL})$. Solution composition: $1 \mathrm{mMFcCOOH}$ in $10 \mathrm{mM}$ PBS. CV scan rate: $100 \mathrm{mVs}^{-1}$. EIS frequency range: $0.1 \mathrm{~Hz}$ to $100 \mathrm{kHz} ; \mathrm{E}=150 \mathrm{mV}$ and $\Delta \mathrm{V}=5 \mathrm{mV}$. 
curve e). These changes may be attributed to the anti-BSA orienting in a manner so as to block the $\mathrm{FcCOOH}$ from reaching the electrode surface preventing electronic transfer to the electrode.

The detection of anti-BSA antibodies $(10 \mu \mathrm{g} / \mathrm{mL})$ in buffer in 20 min in this way demonstrates the potential of these nanowire sensors for use as rapid and label-free immunosensors. To confirm this potential, electrochemical-based detection of BVD antibodies in both buffer and diluted serum samples using virus as capture biomolecules was then assessed.

\section{Detection of BVD antibodies in buffer}

The functionalization process previously described was then applied to the covalent immobilization of BVD virus $(100 \mu \mathrm{g} / \mathrm{mL})$ onto the o-ABA polymer electrodeposited on the nanowire electrode. Figure $4 \mathrm{~A}$ shows a schematic of the layer-by-layer build up on a nanowire surface. The detection of BVD antibodies (BVD Ab) is firstly performed in buffer (PBS; $\mathrm{pH}$ 7.4) for a concentration of $10 \mu \mathrm{g} / \mathrm{mL}$. As previously described, $\mathrm{CV}$ and nyquist spectra were recorded in presence of $\mathrm{FcCOOH}$ as redox probe (Figures $4 \mathrm{~B}$ and $4 \mathrm{C}$ ).

$\mathrm{O}-\mathrm{ABA}$ was again electrodeposited onto pristine gold nanowires (Figure $4 \mathrm{~B}$ and $4 \mathrm{C}$, curve a) leading to a decrease in electron transfer as expected (Figures $4 \mathrm{~B}$ and $4 \mathrm{C}$, curve $\mathrm{b}$ ). BVD virus was then immobilized on the o-ABA modified electrode resulting in an important decrease in the current intensity (Figure $4 \mathrm{~B}$, curve c) and an increase in the semi-circle diameter i.e. increase of the charge transfer resistance and the global capacitance (Figure 4C, curve c). These changes are mainly attributed to the large BVD virus $(40-60 \mathrm{~nm})$, capture molecule, attached on the nanowire preventing $\mathrm{FcCOOH}$ to reach the electrode surface. Following the blocking step, slight changes are visible but the $\mathrm{CV}$ and nyquist spectrum remain very similar to the BVD virus layer (Figures $4 \mathrm{~B}$ and $4 \mathrm{C}$, curve d).

After binding of the antibodies to the multiple epitopes on the peptide envelop of the virus, a strong increase in the current intensity is observed in $\mathrm{CV}$ (Figure 4B, curve e) with a corresponding decrease in the measured impedance (Figure $4 \mathrm{C}$, curve e). This suggests that the binding may cause a re-orientation or re-arrangement of the virus layer forming channels between virus particles through which $\mathrm{FcCOOH}$ molecules may pass to reach the electrode. It also suggests that electronic transfer between BVD antibody and the electrode may be occurring and probably a combination of both.

After successful demonstration of BVD antibodies detection in buffer, the BVD virus modified immunosensor was then applied to detection of BVD antibody in bovine serum of known disease state i.e. with BVD antibody positive and negative, obtained from cows.

\section{Detection of BVD antibodies in serum}

To evaluate the behavior of the electrochemical nanowire-based immunosensor in complex matrices, a serum dilution of $1 \%$ was chosen. This dilution corresponds to the maximum dilution permitting the BVD Antibody detection with the ELISA after $1 \mathrm{~h}$ incubation of the positive infected serum sample. The $\mathrm{CV}$ and nyquist spectra were recorded in presence of $\mathrm{FcCOOH}$ for each step of the virus immobilization and the detection of BDV antibodies in $1 \%$ positive infected serum (Figures 5A and 5B).

As before, immobilization of the BVD virus on the nanowire leads to the limitation of electron transfer of $\mathrm{FcCOOH}$ to the electrode i.e. decrease in current intensity and increase in impedance (Figures $5 \mathrm{~A}$ and $5 \mathrm{~B}$, curves $\mathrm{a}-\mathrm{c}$ ) with no major changes being observed after ethanolamine blocking step (Figures $5 \mathrm{~B}$ and $5 \mathrm{~A}$, curve $\mathrm{d}$ ). The BVD virus immunosensor was then exposed to $1 \%$ positive infected serum incubated for $20 \mathrm{~min}$ and washed to remove any unbound material. The CV and EIS obtained following the binding of BVD antibodies from the positive serum evolve in the same way as in buffer i.e. an increase in the $\mathrm{CV}$ and a decrease in the impedance (Figure $5 \mathrm{~A}$ and
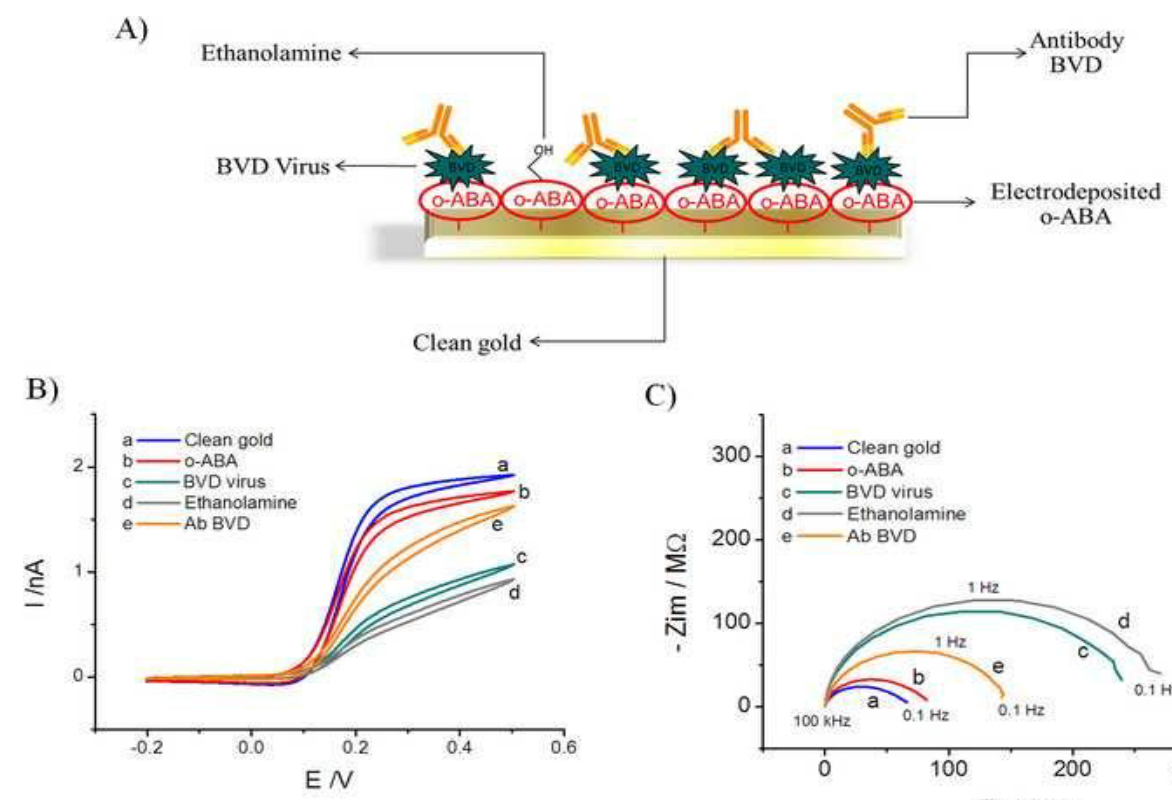

B)
C)

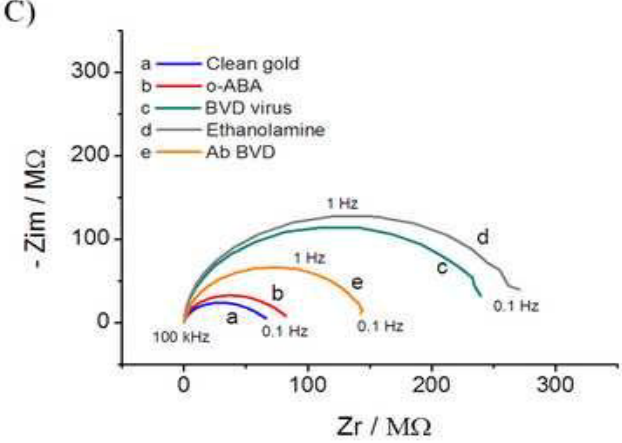

Figure 4: (A) Schematic of the layer-by-layer build up on a nanowire sensor for BVD antibody detection; (B) Cyclic Voltammograms and (C) Nyquist plots of (a) pristine gold nanowire electrode; (b) o-ABA modified electrode; (c) BVD virus (100 $\mu \mathrm{g} / \mathrm{mL}) \mathrm{modified}$ electrode; (d) ethanolamine blocked electrode; (e)Antibody BVD $(10 \mu \mathrm{g} / \mathrm{mL})$ binding in buffer. Solution composition: $1 \mathrm{mMFcCOOH}$ in $10 \mathrm{mM} \mathrm{PBS}$. CV scan rate: $100 \mathrm{mVs}^{-1}$. EIS frequency range: $0.1 \mathrm{~Hz}$ to $100 \mathrm{kHz} ; \mathrm{E}=150 \mathrm{mV}$ and $\Delta \mathrm{V}=5 \mathrm{mV}$. 

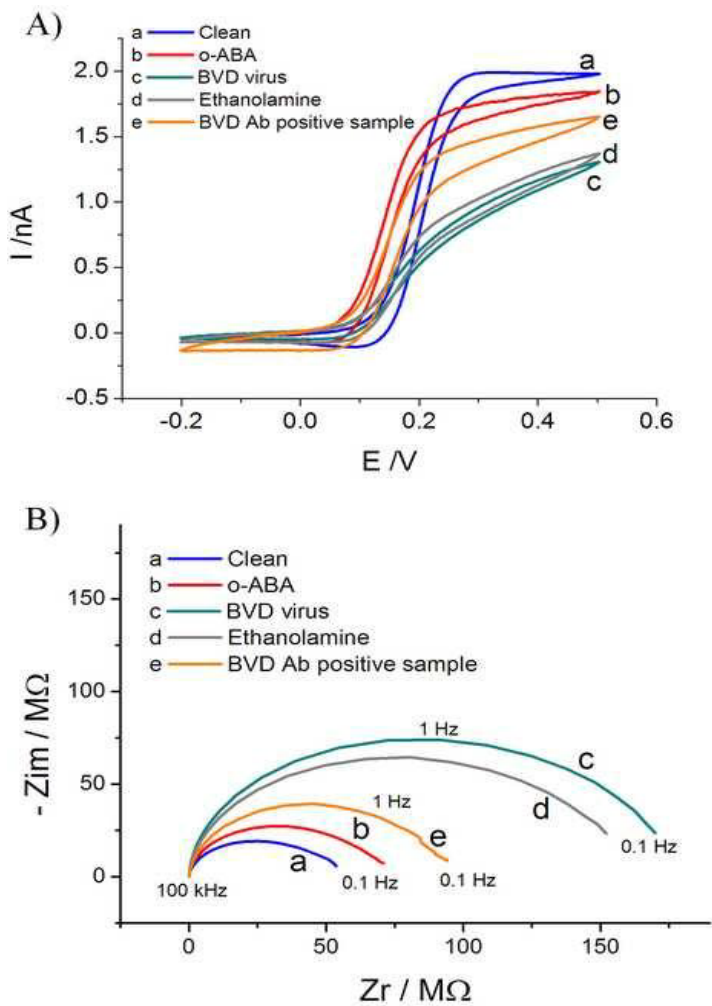

Figure 5: (A) Cyclic Voltammograms and (B) Nyquist plots obtained of (a) pristine gold nanowire electrode;(b) o-ABA modified electrode; (c) BVD virus $(100 \mu \mathrm{g} / \mathrm{mL}$ ) modified electrode; (d) ethanolamine blocked electrode; (e) Antibody BVD binding in $1 \%$ bovine serum positive. Solution composition: 1 $\mathrm{mMFcCOOH}$ in $10 \mathrm{mM}$ PBS. CV scan rate: $100 \mathrm{mVs}^{-1}$. EIS frequency range: $0.1 \mathrm{~Hz}$ to $100 \mathrm{kHz} ; \mathrm{E}=150 \mathrm{mV}$ and $\Delta \mathrm{V}=5 \mathrm{mV}$.


Figure 6: (A) Cyclic Voltammograms and (B) Nyquistplots of (a) pristine gold nanowire electrode; (b) o-ABA modified electrode; (c) BVD virus $(10 \mu \mathrm{g} / \mathrm{mL}$ ) modified electrode; (d) ethanolamine blocked electrode;(e) negative BVD Antibody serum; (f) positive BVD Antibody serum. Solution: $1 \mathrm{mMFcCOOH}$ in $10 \mathrm{mM}$ PBS. CV scan rate: $100 \mathrm{mVs}^{-1}$. EIS frequency range: $0.1 \mathrm{~Hz}$ to $100 \mathrm{kHz}$ $\mathrm{E}=150 \mathrm{mV}$ and $\Delta \mathrm{V}=5 \mathrm{mV}$

5B, curve e). These changes are attributed to immunologic binding of BVD antibodies present in the serum to the virus immobilized on the nanowire.

To confirm these results, control experiments were undertaken using BVD antibody negative serum samples. Following these control experiments, serum positive infected samples aliquots were then dispensed on the sensors to (i) confirm bio-functionality and efficacy of the virus capture probe and (ii) demonstrate the specificity of the sensor against BVD antibodies. Figure 6 presents typical CV and EIS obtained first for BVD antibody negative and then for positive serum samples on the same immunosensor. Figure 6 presents typical CV and
EIS obtained first for BVD antibody negative and then for positive serum samples on the same immunosensor.

A decrease in charge transfer and an increase in capacitance are again observed, following covalent coupling of the BVD virus to the gold nanowire and blocking with ethanolamine, compared to pristine gold nanowire electrode (Figures $6 \mathrm{~A}$ and $6 \mathrm{~B}$, curves a-d). Incubation of negative infected serum sample leads to a decrease in the current intensity (Figure 6A, curve e) and an increase in the impedance (Figure $6 \mathrm{~B}$, curve e) indicating a further reduction of electron transfer (versus the ethanolamine curve). This result is contrary to those observed during BVD Ab binding experiments and suggests the formation of a more insulating layer on the electrode further preventing $\mathrm{FcCOOH}$ molecules from reaching the electrode surface.

Immediately after these assays, BVD antibody positive serum was deposited onto nanowire immunosensor and allowed to incubate for $20 \mathrm{~min}$. An increase in the current intensity and a decrease in the impedance (Figures $6 \mathrm{~A}$ and $6 \mathrm{~B}$, curve $\mathrm{f}$ ), consistent with results presented in Figures 4 and 5, are observed following binding of BVD antibodies present in serum samples to the immobilized virus. These results strongly suggest that the observed changes in both $\mathrm{CV}$ and nyquist spectra are attributed to the presence of BVD antibody in sera sample. Furthermore, it strengthens the arguments of re-orientation or re-arrangement of the virus layer allowing ingress of $\mathrm{FcCOOH}$ molecules to the electrode when BVD antibodies are bound. Finally, this result highlights the fact that immobilized virus are fully functional, not degraded during the experiments and demonstrate the specificity of the sensors to the BVD antibodies. In all cases, electrochemical results were in full agreement with results obtained from the commercial ELISA kit.

The electrochemical based immunologic detection of BVD antibodies in $1 \%$ serum in 20 min demonstrates the suitability of this sensor device for potential use in future on-farm diagnostic devices and opens the door for detection of a wide variety of other diseases.

\section{Conclusion}

This paper presents the first electrochemical-based on-chip nanowire immunosensor device. Using an electrodeposited o-ABA to covalently immobilize the capture biomolecules, electrochemical immunosensing $(10 \mu \mathrm{g} / \mathrm{mL})$ on the on-chip nanowire is validated in buffer using first a model BSA antigen-antibody and then BVD virusantibody. The nanowire-based BDV virus immunosensor allows the specific detection of BDV antibodies in serum in 20 min permitting the discrimination between BVD infected and non-infected bovine sera obtained from cattle. Work is still ongoing to optimize the modification process to increase the performance of the immunosensor.

\section{Acknowlegement}

The authors would like to thank Caoimhe Robinson for undertaking ELISA experiments. This work was supported by Science Foundation Ireland under the US-Ireland "Agri-Sense" project (12/US/I2476) and the Irish Higher Education Authority PRTLI programs (Cycle 3 "Nanoscience" and Cycle 4 "INSPIRE").

\section{References}

1. Ridpath JF (2010) Veterinary Clinics of North America: Food Animal Practice 26: 105-121.

2. Snowder GD, Van Vleck LD, Cundiff LV, Bennett GL, Koohmaraie M, et al (2007) Journal of Animal Science 85: 1885-1892.

3. Dehove A, Commault J, Petitclerc M, Teissier M, Macé J (2012) Economic analysis and costing of animal health: A literature review of methods and importance. Rev Sci Tech 31: 605-617, 591-604.

4. Dubovi EJ (2013) Laboratory diagnosis of bovine viral diarrhea virus 
Citation: Montrose A, Creedon N, Sayers R, Barry S, O'riordan A (2015) Novel Single Gold Nanowire-based Electrochemical Immunosensor for Rapid Detection of Bovine Viral Diarrhoea Antibodies in Serum. J Biosens Bioelectron 6: 174. doi:10.4172/2155-6210.1000174

Biologicals, Aspects of Immunology of Bovine Viral Diarrhea Virus 41: 8-13.

5. Goyal SM (2005) Diagnosis In: Goyal SM, Ridpath JF (eds.). Bovine Viral Diarrhea Virus. Blackwell Publishing Ltd 197-208.

6. Lanyon SR, Hill FI, Reichel MP, Brownlie J (2014) Bovine viral diarrhoea: Pathogenesis and diagnosis. Vet J 199: 201-209.

7. Daniels JS, Pourmand N (2007) Label-free impedance biosensors: Opportunities and Challenges. Electroanalysis 19: 1239-1257.

8. Dolatabadi JEN, Guardia MD (2014) Nanomaterial-based electrochemical immunosensors as advanced diagnostic tools. Analytical Methods 6: 38913900.

9. Chang BY, Park SM (2010) Electrochemical impedance spectroscopy. Annu Rev Anal Chem (Palo Alto Calif) 3: 207-229.

10. Arrigan DW (2004) Nanoelectrodes, nanoelectrode arrays and their applications. Analyst 129: 1157-1165.

11. Barry S, Dawson K, Correa E, Goodacre R, O'Riordan A (2013) Highly sensitive detection of nitroaromatic explosives at discrete nanowire arrays. Faraday Discuss 164: 283-293.

12. Montrose A, Cargou S, Nepveu F, Manczak R, Gué AM, et al. (2013) Impedimetric immunosensor for the detection of circulating pro-inflammatory monocytes as infection markers. Biosens Bioelectron 49: 305-311.

13. Pejcic B, De Marco R (2006) Electrochimica Acta 5: 6217-6229.

14. Godino N, Borrise X, Munoz FX, Del Campo FJ, Compton RG (2009) The Journal of Physical Chemistry 113: 11119-11125.

15. Dawson K, Wahl A, Barry S, Barrett C, Sassiat N, et al. (2014) Fully integrated on-chip nano-electrochemical devices for electroanalytical applications. Electrochimica Acta 115: 239-246.

16. Dawson K, Wahl A, Murphy R, O'Riordan A (2012) Electroanalysis at single gold nanowire electrodes. Journal of Physical Chemistry 116: 14665-14673.

17. Dawson K, Baudequin M, O'Riordan A (2011) Single on-chip gold nanowires for electrochemical biosensing of glucose. Analyst 136: 4507-4513.

18. Wahl A, Dawson K, Sassiat N, Quinn AJ, O'Riordan A (2011) Nanomolar Trace Metal Analysis of Copper at Gold Microband Arrays. Journal of Physics: Conference Series 307: 012-061.

19. Wahl A, Dawson K, MacHale J, Barry S, Quinn AJ, et al. (2013) Gold nanowire electrodes in array: simulation study and experiments. Faraday Discuss 164 : $377-390$
20. Dawson K, Baudequin M, Sassiat N, Quinn AJ, O'Riordan A (2013) Electroanalysis at discrete arrays of gold nanowire electrodes. Electrochimica Acta 101: 169-176.

21. Wahl A, Barry S, Dawson K, MacHale J, Quinn AJ, et al. (2014) Electroanalysis at ultramicro and nanoscale electrodes: A comparative study. Journal of Electrochemical Society 161: B3055-B3060

22. Cui $Y$, Wei Q, Park H, Lieber CM (2001) Nanowire nanosensors for highly sensitive and selective detection of biological and chemical species. Science 293: $1289-1292$

23. Patolsky F, Zheng G, Hayden O, Lakadamyali M, Zhuang X (2004) Electrical detection of single viruses. Proceedings of the National Academy of Sciences 101: 14017-14022.

24. Stern E, Vacic A, Reed MA (2008) Semiconducting Nanowire Field-Effect Transistor Biomolecular Sensors. IEEE Transactions on Electron Devices 55: 3119-3130.

25. Stern E, Klemic JF, Routenberg DA, Wyrembak PN, Turner-Evans DB, et al. (2007) Label-free immunodetection with CMOS-compatible semiconducting nanowires. Nature 445: 519-522.

26. Dawson K, O'Riordan A (2014) Electroanalysis at the nanoscale. Annu Rev Anal Chem (Palo Alto Calif) 7: 163-181.

27. Taleat Z, Cristea C, Marrazza G, Mazloum-Ardakani M, Sandulescu R (2014) Electrochemical immunoassay based on aptamer-protein interaction and functionalized polymer for cancer biomarker detection. Journal of Electroanalytical Chemistry 717-718, 119-124

28. Preechaworapun A, Ivandini TA, Suzuki A, Fujishima A, Chailapakul O, et al. (2008) Development of amperometric immunosensor using boron-doped diamond with poly(o-aminobenzoic acid). Anal Chem 80: 2077-2083.

29. Sriwichai S, Baba A, Phanichphant S, Shinbo K, Kato K, et al. (2010) Electrochemically controlled surface plasmon resonance immunosensor for the detection of human immunoglobulin $\mathrm{G}$ on poly(3-aminobenzoic acid) ultrathin films. Sensors and Actuators, Chemical 147: 322-329.

30. Mazloum-Ardakani M, Hosseinzadeh L, Taleat Z (2014) Microchimica Acta 18 917-924.

31. Wang Y, Knoll W (2006) In situ electrochemical and surface plasmon resonance (SPR) studies of aniline-carboxylated aniline copolymers. Analytica Chimica Acta 558: 150-157 